January 2020

\title{
Graham's Pebbling Conjecture Holds for the Product of a Graph and a Sufficiently Large Complete Graph
}

Nopparat Pleanmani

Khon Kaen University, kokho30@gmail.com

Follow this and additional works at: https://digitalcommons.georgiasouthern.edu/tag

Part of the Discrete Mathematics and Combinatorics Commons

\section{Recommended Citation}

Pleanmani, Nopparat (2020) "Graham's Pebbling Conjecture Holds for the Product of a Graph and a Sufficiently Large Complete Graph," Theory and Applications of Graphs: Vol. 7 : Iss. 1 , Article 1. DOI: $10.20429 /$ tag.2020.070101 Available at: https://digitalcommons.georgiasouthern.edu/tag/vol7/iss1/1

This article is brought to you for free and open access by the Journals at Digital Commons@Georgia Southern. It has been accepted for inclusion in Theory and Applications of Graphs by an authorized administrator of Digital Commons@Georgia Southern. For more information, please contact digitalcommons@georgiasouthern.edu. 
Graham's Pebbling Conjecture Holds for the Product of a Graph and a Sufficiently Large Complete Graph

\section{Cover Page Footnote}

The authors would like to thank the referee(s) for comments and suggestions on the manuscript. This research was supported by Development and Promotion of Science and technology Talents project (DPST). 


\begin{abstract}
For connected graphs $G$ and $H$, Graham conjectured that $\pi(G \square H) \leq \pi(G) \pi(H)$ where $\pi(G), \pi(H)$, and $\pi(G \square H)$ are the pebbling numbers of $G, H$, and the Cartesian product $G \square H$, respectively. In this paper, we show that the inequality holds when $H$ is a complete graph of sufficiently large order in terms of graph parameters of $G$.
\end{abstract}

\title{
1 Introduction
}

Throughout this paper, all graphs are considered to be finite and simple. For a graph $G$, we denote the order of $G$ by $|G|$. For a positive integer $n$, we denote $K_{n}$ to be a complete graph of $n$ vertices. For basic definitions and terminologies not mentioned here, we refer the reader to the book of West [10].

Given two graphs $G$ and $H$, the Cartesian product of $G$ and $H$, denoted by $G \square H$, is the graph with the vertex set $V(G) \times V(H)$ and the edge set

$\left\{\left(u, v_{1}\right)\left(u, v_{2}\right): u \in V(G)\right.$ and $\left.v_{1} v_{2} \in E(H)\right\} \cup\left\{\left(u_{1}, v\right)\left(u_{2}, v\right): u_{1} u_{2} \in E(G)\right.$ and $\left.v \in V(H)\right\}$.

We note that $G \square H$ is connected if and only if $G$ and $H$ are both connected. For more detail treatments of graph products, we refer the reader to [7]. In order to study graph products practically, we need some definitions that consider the product of sets $A$ and $B$. In particular, if $C \subseteq A \times B$, we define $p_{1}(C)=\{a:(a, b) \in C$ where $b \in B\}$. For a function $f$ from a finite set $I$ to the set $\mathbb{N} \cup\{0\}$, we recall that $\sum_{i \in I} f(i)=0$ whenever $I=\emptyset$. And we use this convention for Lemma 2.1 and the proof of Proposition 2.1. Moreover, for graphs $G$ and $H$, we denote $S \square H$ and $G \square T$ the induced subgraphs of $G \square H$ induced by $S \times V(H)$ and $V(G) \times T$, respectively, where $S \subseteq V(G)$ and $T \subseteq V(H)$.

Let $G$ be a connected graph. A (pebbling) configuration on $G$ is defined to be a function $D: V(G) \rightarrow \mathbb{N} \cup\{0\}$ or we can say that $D$ distributes $\sum_{v \in V(G)} D(v)$ pebbles on $G$. A configuration $D$ on $G$ is said to be moveable if there exist two adjacent vertices $u$ and $v$ such that $D(u) \geq 2$. For a moveable configuration $D$ on a graph $G$ and adjacent vertices $u$ and $v$ with $D(v) \geq 2$, the (pebbling) move from $u$ to $v$ in $G$ is defined to be the triple $(D, u, v)$ and we denote it by $D(u \rightarrow v)$ for convenience. For a move $D(u \rightarrow v)$ in $G$, the configuration $D^{\prime}: V(G) \rightarrow \mathbb{N} \cup\{0\}$ defined by

$$
D^{\prime}(x)= \begin{cases}D(x)-2 & \text { if } x=u \\ D(x)+1 & \text { if } x=v \\ D(x) & \text { otherwise }\end{cases}
$$

is called the configuration with respect to $D(u \rightarrow v)$. Let $D$ be a moveable configuration on a graph $G$. A $D$-moving sequence in $G$ is a finite sequence of moves $D_{1}\left(u_{1} \rightarrow v_{1}\right), D_{2}\left(u_{2} \rightarrow\right.$ $\left.v_{2}\right), \ldots, D_{n}\left(u_{n} \rightarrow v_{n}\right)$ such that $D=D_{1}$ and $D_{i}$ is the moveable configuration with respect to $D_{i-1}\left(u_{i-1} \rightarrow v_{i-1}\right)$ for every $i \in\{2, \ldots, n\}$ and we write $u_{1} \rightarrow v_{1}, u_{2} \rightarrow v_{2}, \ldots, u_{n} \rightarrow v_{n}$ for convenience. For a vertex $r$ of $G$, if $r$ appears in some $D$-moving sequences or $D(r) \geq 1$, we say that one can pebble $r$ under a configuration $D$ on $G$ or we can say that $D$ is $r$-solvable on $G$. Furthermore, a configuration is solvable whenever it is $r$-solvable for every vertex 
$r$. It is unsolvable otherwise. Given a configuration $D$ on a connected graph $G$; we call $\sum_{v \in V(G)} D(v)$ the size of $D$ and denoted by $|D|$. In a Cartesian product graph $G \square H,\left|D_{x}\right|$ denotes $\sum_{v \in V(H)} D(x, v)$ for each $x \in V(G)$. The pebbling number of a connected graph $G$, denoted by $\pi(G)$, is the smallest integer $m$ such that $D$ is solvable for every configuration $D$ on $G$ with $|D| \geq m$. We note a basic fact, mentioned by Chung [1], of pebbling number of a connected graph $G$ that $\pi(G) \geq|G|$. For a survey of graph pebbling we refer the reader to [5], [6] and [8]. Now, we introduce a new graph pebbling parameter called the support number which is actually an extension of the pebbling number. The support of a configuration $D$ on a connected graph $G$ means the set $\{v \in V(G): D(v)>0\}$. For a connected graph $G$ and a positive integer $n$, the $n$-support number of $G$ is the minimum $m$ such that $D$ is solvable for any configuration $D$ on $G$ with $\sum_{v \in V(G)}\left\lfloor\frac{D(v)}{n}\right\rfloor \geq m$ if $n \leq \pi(G)$. It equals 1 otherwise. Obviously, the 1-support number is actually the pebbling number. Additionally, we denote the 2-support number of $G$ by $\tilde{\pi}(G)$.

One of the interesting topics in recent graph pebbling is the Graham's conjecture which introduced by Chung [1]. It is about an upper bound of the pebbling number of the Cartesian product of graphs as follows:

Conjecture 1.1. [1] If $G$ and $H$ are connected, then

$$
\pi(G \square H) \leq \pi(G) \pi(H) .
$$

Chung [1] showed that the conjecture holds when $H$ is a complete graph and $G$ is a graph satisfying the so-called 2-pebbling property. Such property plays an important role in verifying the conjecture for certain families of graphs. In case $H$ is a complete graph, it is in general still open by Herscovici [4]. However, we make progress toward this work from a different perspective by focusing on the order of the complete graph $H$ in terms of $\pi(G)$ and $|G|$ as we see in the next section.

\section{Main Results}

In this section, we will prove Theorem 2.4 by means of the technical Lemma 2.2 about the 2-support number.

Lemma 2.1. Let $G=(V, E)$ be a connected graph, $S$ be a subset of $V$ and $D$ be a configuration on $G$. Then we have

$$
\sum_{v \in V \backslash S} D(v)-n \sum_{v \in V \backslash S}\left\lfloor\frac{D(v)}{n}\right\rfloor \leq(n-1)(|G|-|S|)
$$

for any positive integer $n$.

Proof. The inequality holds since $D(v)-n\left\lfloor\frac{D(v)}{n}\right\rfloor \leq n-1$ for each $v \in V$.

We see that the configuration $D$ on $G$ defined by $D(v)=n-1$ for each $v \in V$ attains the upper bound in Lemma 2.1. 
Lemma 2.2. For a nontrivial connected graph $G$ and a positive integer $m$ greater than 1 , we have

$$
\tilde{\pi}\left(G \square K_{m}\right) \leq \pi(G)
$$

Proof. Let $V^{\prime}=V\left(G \square K_{m}\right), D$ be a configuration on $G \square K_{m}$ with $\sum_{(x, y) \in V^{\prime}}\left\lfloor\frac{D(x, y)}{2}\right\rfloor \geq \pi(G)$ and $(r, t)$ be a vertex of $G \square K_{m}$. Let $M=\left\{(x, y) \in V^{\prime}: D(x, y)>1\right\}$ and let $M_{x}=$ $\left\{z \in V\left(K_{m}\right):(x, z) \in M\right\}$ for each $x \in p_{1}(M)$. Then we can pebble $(x, t)$ with at least $\sum_{z \in M_{x}}\left\lfloor\frac{D(x, z)}{2}\right\rfloor$ pebbles for each $x \in p_{1}(M)$ since $\tilde{\pi}\left(K_{m}\right)=1$. Let $D^{\prime}$ be a configuration on $G \square K_{m}$ after pebbling $(x, t)$ with at least $\sum_{z \in M_{x}}\left\lfloor\frac{D(x, z)}{2}\right\rfloor$ pebbles for all $x \in p_{1}(M)$. It follows that

$$
\begin{aligned}
\sum_{x \in V(G)} D^{\prime}(x, t) & \geq \sum_{x \in p_{1}(M)} D^{\prime}(x, t) \geq \sum_{x \in p_{1}(M)} \sum_{z \in M_{x}}\left\lfloor\frac{D(x, z)}{2}\right\rfloor \\
& =\sum_{(x, y) \in V^{\prime}}\left\lfloor\frac{D(x, y)}{2}\right\rfloor \geq \pi(G) .
\end{aligned}
$$

Hence we can pebble $(r, t)$ within the induced subgraph $G \square\{t\}$.

Lemma 2.3. Let $G$ be a nontrivial connected graph with $V=V(G)$. For a positive integer $n$, let $D$ be a configuration on $G \square K_{n}$ and $(r, t)$ be a vertex of $G \square K_{n}$. If $S$ is a proper subset of $V$ containing $r$ such that $\sum_{x \in V \backslash S}\left|D_{x}\right| \geq n(|V \backslash S|)+2 \pi(G)$, then one can pebble $(r, t)$.

Proof. Let $V^{\prime}=V\left(G \square K_{n}\right)$ and $S^{\prime}=V\left(S \square K_{n}\right)$. By Lemma 2.1, we obtain that

$$
\begin{aligned}
2 \sum_{(x, y) \in V^{\prime} \backslash S^{\prime}}\left\lfloor\frac{D(x, y)}{2}\right\rfloor & \geq\left(\sum_{(x, y) \in V^{\prime} \backslash S^{\prime}} D(x, y)\right)-\left(\left|V^{\prime}\right|-\left|S^{\prime}\right|\right) \\
& =\left(\sum_{x \in V \backslash S}\left|D_{x}\right|\right)-(n|G|-n|S|)=\left(\sum_{x \in V \backslash S}\left|D_{x}\right|\right)-n(|G|-|S|) \\
& =\left(\sum_{x \in V \backslash S}\left|D_{x}\right|\right)-n|V \backslash S| \geq n|V \backslash S|+2 \pi(G)-n|V \backslash S| \\
& =2 \pi(G) .
\end{aligned}
$$

By Lemma 2.2,

$$
\sum_{(x, y) \in V^{\prime}}\left\lfloor\frac{D(x, y)}{2}\right\rfloor \geq \sum_{(x, y) \in V^{\prime} \backslash S^{\prime}}\left\lfloor\frac{D(x, y)}{2}\right\rfloor \geq \pi(G) \geq \tilde{\pi}\left(G \square K_{n}\right) .
$$

Therefore, we can pebble $(r, t)$.

Now, we are ready for determining an upper bound for the pebbling number of the Cartesian product of a graph and a complete graph. 
Proposition 2.1. For a positive integer $n$ and a connected graph $G$, we have

$$
\pi\left(G \square K_{n}\right) \leq n|G|+2 \pi(G)-2 .
$$

Proof. Let $V=V(G)$ and $V^{\prime}=V\left(K_{n}\right)$. If $\left|D_{r}\right| \geq n$, then we can pebble $(r, t)$. In addition, we can assume that $\left|D_{r}\right| \leq n-1$. We now consider the following two cases.

Case 1: $\left|D_{r}\right| \leq n-2$.

Clearly,

$$
\begin{aligned}
\sum_{x \in V \backslash\{r\}}\left|D_{x}\right| & =|D|-\left|D_{r}\right| \geq|D|-(n-2)=(n|G|+2 \pi(G)-2)-(n-2) \\
& =n(|G|-1)+2 \pi(G)=n|V \backslash\{r\}|+2 \pi(G) .
\end{aligned}
$$

By Lemma 2.3, we can pebble $(r, t)$.

Case 2: $\left|D_{r}\right|=n-1$.

If $D\left(r, v_{r}\right) \geq 2$ for some $v_{r} \in V^{\prime} \backslash\{t\}$, then we can pebble $(r, t)$. So we can assume that $D\left(r, v_{r}\right)=1$ for all $v_{r} \in V^{\prime} \backslash\{t\}$. Since $n(|G|-1)+2 \pi(G)-1 \geq n(|G|-1)+1$, there are at least $n(|G|-1)+1$ pebbles distributed by $D$ on $n(|G|-1)$ vertices in $V\left(G \square K_{n}\right) \backslash V\left(\{r\} \square K_{n}\right)$. By the pigeonhole principle, $D(g, u) \geq 2$ for some $(g, u) \in V\left(G \square K_{n}\right) \backslash V\left(\{r\} \square K_{n}\right)$. Let $g=w_{1}, w_{2}, \ldots, w_{m}=r$ be a $g, r$-path in $G$. Obviously, $m \geq 2$ since $g \neq r$. Note that

$$
\begin{aligned}
\sum_{x \in V \backslash\left\{w_{m}\right\}}\left|D_{x}\right| & =\sum_{x \in V \backslash\{r\}}\left|D_{x}\right|=|D|-\left|D_{r}\right|=|D|-(n-1) \\
& =(n|G|+2 \pi(G)-2)-(n-1)=n(|G|-1)+2 \pi(G)-1 .
\end{aligned}
$$

This implies that $V \backslash\left\{w_{m}\right\} \neq \emptyset$ since $n(|G|-1)+2 \pi(G)-1 \geq 2 \pi(G)-1>0$. In this case, we can succeed within $m-1$ steps.

\section{Step 1.}

If $D\left(w_{m-1}, v_{m-1}\right) \geq 2$ for some $v_{m-1} \in V^{\prime}$, then we move

- $\left(w_{m-1}, v_{m-1}\right) \rightarrow\left(w_{m}, t\right)=(r, t)$ if $v_{m-1}=t$;

- $\left(w_{m-1}, v_{m-1}\right) \rightarrow\left(w_{m}, v_{m-1}\right),\left(w_{m}, v_{m-1}\right) \rightarrow\left(w_{m}, t\right)=(r, t)$ if $v_{m-1} \neq t$.

In addition, we can assume that $D\left(w_{m-1}, v_{m-1}\right) \leq 1$ for all $v_{m-1} \in V^{\prime}$, i.e., $\left|D_{w_{m-1}}\right| \leq n$.

- If $D\left(w_{m-1}, v_{m-1}\right)=1$ for all $v_{m-1} \in V^{\prime}$, then $\left|D_{w_{m-1}}\right|=n$ and so

$$
\begin{aligned}
\sum_{x \in V \backslash\left\{w_{m-1}, w_{m}\right\}}\left|D_{x}\right| & =\left(\sum_{x \in V \backslash\left\{w_{m}\right\}}\left|D_{x}\right|\right)-\left|D_{m-1}\right| \\
& =\left(\sum_{x \in V \backslash\left\{w_{m}\right\}}\left|D_{x}\right|\right)-n \\
& =(n(|G|-1)+2 \pi(G)-1)-n \\
& =n(|G|-2)+2 \pi(G)-1 .
\end{aligned}
$$

This implies $V \backslash\left\{w_{m-1}, w_{m}\right\} \neq \emptyset$ since $\sum_{x \in V \backslash\left\{w_{m-1}, w_{m}\right\}}\left|D_{x}\right| \geq n(|G|-2)+2 \pi(G)-1 \geq$ $2 \pi(G)-1 \geq 2|G|-1>0$. So $|G| \geq 3$ and we go to Step 2 . 
- If $D\left(w_{m-1}, v_{m-1}\right)=0$ for some $v_{m-1} \in V^{\prime}$, then $\left|D_{w_{m-1}}\right| \leq n-1$ and so

$$
\begin{aligned}
\sum_{x \in V \backslash\left\{w_{m-1}, w_{m}\right\}}\left|D_{x}\right| & =\left(\sum_{x \in V \backslash\left\{w_{m}\right\}}\left|D_{x}\right|\right)-\left|D_{w_{m-1}}\right| \\
& \geq\left(\sum_{x \in V \backslash\left\{w_{m}\right\}}\left|D_{x}\right|\right)-(n-1) \\
& =(n(|G|-1)+2 \pi(G)-1)-(n-1) \\
& =n(|G|-2)+2 \pi(G) \\
& =n\left|V \backslash\left\{w_{m-1}, w_{m}\right\}\right|+2 \pi(G) .
\end{aligned}
$$

By Lemma 2.3, we can pebble $(r, t)$.

Step $\mathbf{i}(1 \leq \mathrm{i} \leq m-2)$.

If $D\left(w_{m-i}, v_{m-i}\right) \geq 2$ for some $v_{m-i} \in V^{\prime}$, then we move

- $\left(w_{m-i}, v_{m-i}\right) \rightarrow\left(w_{m-i+1}, v_{m-i}\right), \ldots,\left(w_{m-2}, v_{m-i}\right) \rightarrow\left(w_{m-1}, v_{m-i}\right),\left(w_{m-1}, v_{m-i}\right) \rightarrow$ $\left(w_{m}, t\right)=(r, t)$ if $v_{m-i}=t$

- $\left(w_{m-i}, v_{m-i}\right) \rightarrow\left(w_{m-i+1}, v_{m-i}\right), \ldots,\left(w_{m-2}, v_{m-i}\right) \rightarrow\left(w_{m-1}, v_{m-i}\right),\left(w_{m-1}, v_{m-i}\right) \rightarrow$ $\left(w_{m}, v_{m-i}\right),\left(w_{m}, v_{m-i}\right) \rightarrow\left(w_{m}, t\right)=(r, t)$ if $v_{m-i} \neq t$.

In addition, we can assume that $D\left(w_{m-i}, v_{m-i}\right) \leq 1$ for all $v_{m-i} \in V^{\prime}$, i.e., $\left|D_{w_{m-i}}\right| \leq n$.

- If $D\left(w_{m-i}, v_{m-i}\right)=1$ for all $v_{m-i} \in V^{\prime}$, then $\left|D_{w_{m-i}}\right|=n$ and so

$$
\begin{aligned}
\sum_{x \in V \backslash\left\{w_{m-i}, \ldots, w_{m-1}, w_{m}\right\}}\left|D_{x}\right| & =\left(\sum_{x \in V \backslash\left\{w_{m-i+1}, \ldots, w_{m-1}, w_{m}\right\}}\left|D_{x}\right|\right)-\left|D_{w_{m-1}}\right| \\
& =\left(\sum_{x \in V \backslash\left\{w_{m-i+1}, \ldots, w_{m-1}, w_{m}\right\}}\left|D_{x}\right|\right)-n \\
& =(n(|G|-i)+2 \pi(G)-1)-n \\
& =n(|G|-(i+1))+2 \pi(G)-1 .
\end{aligned}
$$

This implies $V \backslash\left\{w_{m-i}, \ldots, w_{m-1}, w_{m}\right\} \neq \emptyset$ since $\sum_{x \in V \backslash\left\{w_{m-i}, \ldots, w_{m-1}, w_{m}\right\}}\left|D_{x}\right| \geq n(|G|-$ $(i+1))+2 \pi(G)-1 \geq 2 \pi(G)-1 \geq 2|G|-1>0$. So $|G| \geq i+2$ and we go to Step $\mathrm{i}+1$. 
- If $D\left(w_{m-i}, v_{m-i}\right)=0$ for some $v_{m-i} \in V^{\prime}$, then $\left|D_{w_{m-i}}\right| \leq n-1$ and so

$$
\begin{aligned}
\sum_{x \in V \backslash\left\{w_{m-i}, \ldots, w_{m-1}, w_{m}\right\}}\left|D_{x}\right| & =\left(\sum_{x \in V \backslash\left\{w_{m-i+1}, \ldots, w_{m-1}, w_{m}\right\}}\left|D_{x}\right|\right)-\left|D_{w_{m-i}}\right| \\
& \geq\left(\sum_{x \in V \backslash\left\{w_{m-i+1}, \ldots, w_{m-1}, w_{m}\right\}}\left|D_{x}\right|\right)-(n-i) \\
& =(n(|G|-i)+2 \pi(G)-1)-(n-i) \\
& =n(|G|-(i+1))+2 \pi(G) \\
& =n\left|V \backslash\left\{w_{m-i}, \ldots, w_{m-1}, w_{m}\right\}\right|+2 \pi(G) .
\end{aligned}
$$

By Lemma 2.3, we can pebble $(r, t)$.

\section{Step m-1.}

Since $D\left(w_{1}, u\right)=D(g, u) \geq 2$, we can move

- $\left(w_{1}, u\right) \rightarrow\left(w_{2}, u\right), \ldots,\left(w_{m-2}, u\right) \rightarrow\left(w_{m-1}, u\right),\left(w_{m-1}, u\right) \rightarrow\left(w_{m}, u\right)=(r, t)$ if $u=t$;

- $\left(w_{1}, u\right) \rightarrow\left(w_{2}, u\right), \ldots,\left(w_{m-2}, u\right) \rightarrow\left(w_{m-1}, u\right),\left(w_{m-1}, u\right) \rightarrow\left(w_{m}, u\right),\left(w_{m}, u\right) \rightarrow\left(w_{m}, t\right)$ $=(r, t)$ if $u \neq t$.

It is easy to establish the sharpness of the upper bound stated in Proposition 2.1, by considering $G=K_{1}$ together with the fact that $\pi\left(K_{1} \square K_{n}\right)=\pi\left(K_{n}\right)=n$.

In the following result, we obtain an alternative sufficient condition for the Cartesian product of a graph and a complete graph to satisfy Graham's conjecture.

Theorem 2.4. For a positive integer $n$ and a connected graph $G$, if $\pi(G)>|G|$ and $n \geq \frac{2(\pi(G)-1)}{\pi(G)-|G|}$, then

$$
\pi\left(G \square K_{n}\right) \leq \pi(G) \pi\left(K_{n}\right) .
$$

Proof. If $\pi(G)>|G|$ then $n \geq \frac{2(\pi(G)-1)}{\pi(G)-|G|}$ implies $n|G|+2 \pi(G)-2 \leq n \pi(G)=\pi\left(K_{n}\right) \pi(G)$ so the results follows from Proposition 2.1.

We note that the condition in Theorem 2.4 does not imply the 2-pebbling property of $G$ as one can see in the following counter example. For a positive integer $k$, Gao and Yin [2] not only proved that the graph $L_{k}$ (see Fig. 1) does not satisfy the 2-pebbling property, but they also showed that $\pi\left(L_{k}\right)=2^{k+3}$.

However, $L_{k}$ satisfies the condition of $G$ in Theorem 2.4 for each $k$ with a sufficiently large $n$. And we obtain the following partial result of Gao and Yin [3].

Corollary 2.5. For positive integers $k$ and $n$, if $\frac{2}{n}+\frac{4 k+7}{2^{k+3}-1} \leq 1$, then

$$
\pi\left(L_{k} \square K_{n}\right) \leq \pi\left(L_{k}\right) \pi\left(K_{n}\right) .
$$

for positive

Proof. By mathematical induction on $k, \pi\left(L_{k}\right)=2^{k+3}>4 k+8=\left|L_{k}\right|$. Furthermore, we can derive $\frac{2}{n}+\frac{4 k+7}{2^{k+3}-1} \leq 1$ from $n \geq \frac{2\left(\pi\left(L_{k}\right)-1\right)}{\pi\left(L_{k}\right)-\left|L_{k}\right|}=\frac{2\left(2^{k+3}-1\right)}{2^{k+3}-4 k-8}$. Hence the result follows by Theorem 2.4 . 


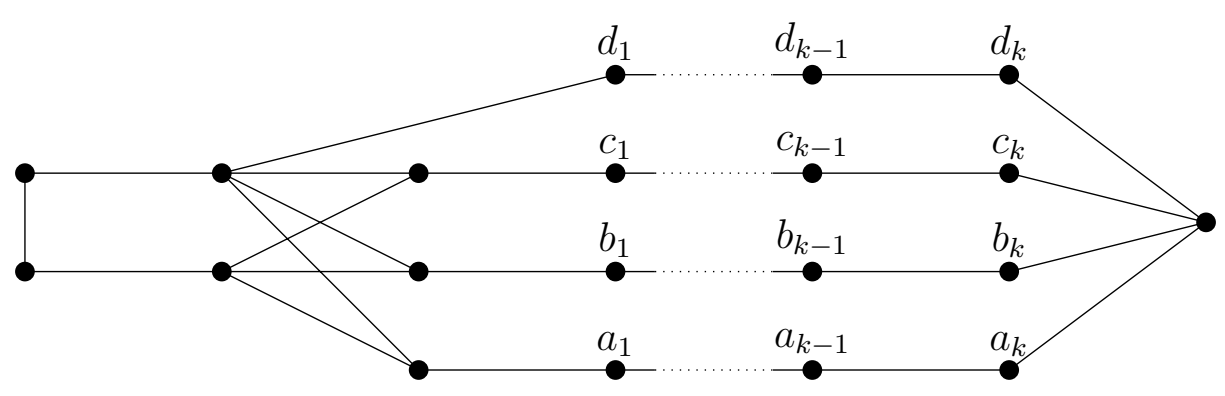

Figure 1: The graph $L_{k}$.

\section{References}

[1] F. Chung. Pebbling in hypercubes. SIAM J. Disc. Math., 2:467-472, 1989.

[2] Z.-T. Gao, and J.-H. Yin. The proof of a conjecture due to Snevily. Discrete Math., 310:1614-1621, 2010.

[3] Z.-T. Gao, and J.-H. Yin. Lemke graphs and Graham's pebbling conjecture. Discrete Math., 340:2318-2332, 2017.

[4] D.S. Herscovici. On graph pebbling numbers and Graham's conjecture. Graph Theory Notes of New York, 59:15-21, 2010.

[5] G. Hurlbert. Graph pebbling. In J. L. Gross, J. Yellen, and P. Zhang, editors. Handbook of graph theory, CRC Press, 2nd edition, 2014.

[6] G. Hurlbert. The graph pebbling page. http://www.people.vcu.edu/ ghurlbert/ pebbling/pebb.html.

[7] W. Imrich, and S. Klavzar Product graphs, structure and recognition, Wiley-Interscience, New York, 2000.

[8] M. Mohorn. An introduction to graph pebbling. Thesis, Davidson College. May 2014.

[9] S.S. Wang. Pebbling and Graham's conjecture. Discrete Math., 226:431-438, 2001.

[10] D.B. West. Introduction to graph theory, Prentice Hall, Upper Saddle River, NJ, 1996. 